\title{
The influence of mouth breathing on skeletal and dental features of splanchnocranium
}

\author{
Lysy $\mathrm{J}^{1}$, Karkazi $\mathrm{F}^{2}$, Stanko $\mathrm{P}^{1}$, Novak $\mathrm{B}^{1}$ \\ Department of Stomatology and Maxilofacial Surgery, Comenius University in Bratislava, \\ Bratislava, Slovakia. jurajlysy@gmail.com
}

\begin{abstract}
OBJECTIVE: This study was performed to investigate changes in dentofacial characteristics associated with mouth breathing (MB) and adenoidectomy.

BACKGROUND: MB is considered to be an etiological factor of malocclusion. Adenoidectomy is supposed to have the ability to prevent the development of dentofacial deformities.

METHODS: This retrospective study included 123 patients, namely 57 nose breathers, 19 former mouth breathers, who have undergone adenoidectomy, and 47 mouth breathers. The groups were compared according to their skeletal and dental characteristics. The measurements of each individual were obtained from lateral cephalograms and dental casts. The comparison was done using one-way ANOVA, Bonferroni post-hoc, Mann-Whitney $U$ and Kruskal-Wallis tests. The statistically significant difference was defined as $p<0.05$.

RESULTS: The MB group showed an increase in ArGoMe $(p=0.02)$ angle. No difference was found in the sagittal parameters among the groups. Upper dental arch compression was positively correlated with MB $(p=0.00)$, even in adenoidectomy cases $(p=0.01)$.

CONCLUSION: MB alters the vertical and transverse growth of the craniofacial complex. It is associated with longer lower anterior facial height and decreased maxillary intermolar distance. However, it does not influence the sagittal parameters. Airway clearance via adenoidectomy promotes the normalization of vertical parameters (Tab. 1, Fig. 2, Ref. 20). Text in PDF www.elis.sk

KEY WORDS: mouth breathing, malocclusion, craniofacial growth, adenoidectomy, cephalometrics.
\end{abstract}

\section{Introduction}

Nasal breathing in coordination with normal swallowing, mastication, posture of the head, tongue, and lips promotes the proper growth and development of splanchnocranium (1). Therefore, any dysfunction can result in aberrant skeletal and dental characteristics of the craniofacial complex. Clinical and experimental data have shown that adjacent soft tissues influence the growth. This principle is known as "functional matrix theory" and was firstly stated by Moss (2). Even light forces can determine the growth pattern of splanchnocranium if they are of long duration. Therefore, since mouth breathing disturbs the equilibrium of oropharyngeal muscles, it causes muscular and postural alterations (3).

Chronic upper airway obstruction affects negatively the development of craniofacial complex. It has been mostly associated with adenoid hypertrophy, thus the term "adenoid faces" (4), which is synonymous with the "long face syndrome" and the "high angle" facial pattern (5). The typical features of mouth breathers include increased lower facial height, lip incompetence, narrow alar base, compressed maxillary dental arch with high palatal vault, posterior

${ }^{1}$ Department of Stomatology and Maxilofacial Surgery, Comenius University in Bratislava, Bratislava, Slovakia, and ${ }^{2}$ Department of Orthodontics, Marmara University, Istanbul, Turkey

Address for correspondence: J. Lysy, MD, PhD, Department of Stomatology and Maxilofacial Surgery, Comenius University in Bratislava, Heydukova 8, SK-812 50 Bratislava, Slovakia. Phone: +421.949477805 crossbite, and class II skeletal pattern (6). Mouth-breathing patients have mixed breathing. Nowadays, exclusive mouth breathing is infrequent owing to the development of anti-allergic drugs and surgical procedures (7).

Although there is significant evidence that deficient nose breathing leads to mouth-nose breathing, its exact impact on splanchnocranium morphology remains obscure. Therefore, there is an unequivocal necessity for further investigation. This study was aimed at assessing the differences in skeletal and dental characteristics of the splanchnocranium through lateral cephalometric radiographs and dental casts between nose-breathing, adenoidectomy, and mouth-breathing patients.

The removal of obstructive factors is considered to promote the normalization of breathing patterns, which thus balances the dentition growth and enhances the orthodontic treatment stability (8). Hence, it has been proposed that adenoidectomy or tonsillectomy could potentially inhibit or even reverse the development of dentofacial deformity at early stages of skeletal development (9).

\section{Materials and methods}

\section{Study design}

This observational retrospective study was approved by the Ethical Committee of St. Elizabeth Cancer Institute, Heydukova 10, 812 50, Bratislava, Slovakia. The study involved 123 patients (72 females and 51 males) aged between 9 and 47 years, who sought 
orthodontic treatment at the Department of Stomatology and Maxillofacial Surgery, Comenius University in Bratislava. Informed consents were obtained from all patients or legal guardians. The sample was consecutive and consisted of a large number of subjects to prevent control errors, and the same examiner performed all the data measurements to ensure consistency and quality of results $(10,11)$.

Based on the anamnestic data and clinical examinations, the patients were divided into three groups according to their predominant breathing type and history of adenoidectomy. The first group included nasal-breathing (NB) patients (35 females and 22 males; age $15.08 \pm 7.36$ years). The second group was composed of former mouth-breathing (FMB) patients (14 females and 5 males; age of $20 \pm 11.62$ years). This group had a positive medical history of adenoid hypertrophy and mouth breathing. Subsequently, they underwent adenoidectomy at age 3 to 6 years to establish nose breathing. The third group consisted of mouth-breathing (MB) patients (23 females and 24 males; age $13.04 \pm 4.76$ ). The FMB and MB groups were considered as study groups and the NB group served as a control. Patients who reported to have developed bad habits (eg. thumb sucking, pacifier use, tongue thrusting) and those who had been already treated orthodontically were excluded.

\section{Cephalometric image and dental cast analysis}

Digital lateral cephalometric radiographs and alginate impressions were obtained for all the patients; the latter were processed on the same day to create plaster study models. One of the authors, who was blinded to the patients' respiration pattern and their adenoidectomy history, performed the evaluations of the dental and skeletal characteristics. The lateral cephalometric analysis was obtained to calculate the sagittal and vertical skeletal parameters, whereas, the values of plaster models were used to evaluate the transverse parameters. More specifically, the intercanine, intepremolar, and intermolar widths were measured with a digital calliper. Ectopic or significantly anomalous teeth were excluded from the cast analysis. Further, all data were numerically labelled and sent to another author, who was also blinded to group allocation of patients, to perform the statistical analysis.

\section{Cephalometric analysis}

The following cephalometric variables were evaluated:

Sagittal parameters (clinical norm):

ANB $\left(1.6 \pm 1.5^{\circ}\right)$ - represents the relative position of the maxilla to mandible

SNA $\left(82.0 \pm 3.5^{\circ}\right)$ - represents the sagittal position of the maxilla relative to the cranial base

$\operatorname{SNB}\left(80.0 \pm 3.0^{\circ}\right)$ - represents the sagittal position of the mandible relative to the cranial base

Vertical parameters (clinical norm):

PP-MP $\left(25 \pm 6^{\circ}\right)$ - represents the palatal plane relative to the mandibular plane

SN-MP $\left(33 \pm 6^{\circ}\right)$ - represents the anterior cranial base relative to the mandibular plane.

$\mathrm{N}-\mathrm{S}$-Ba $\left(131 \pm 4.5^{\circ}\right)$ - describes the cranial base flexure angle

ArGoMe $\left(124.9 \pm 6.7^{\circ}\right)$ - describes the degree inclination of the ramus relative to the body of the mandible.

\section{Model analysis:}

Maxilla (Mx):

- Intercanine distance (MxIC)

- Distance between the buccal cusps of first premolars (MxIP)

- Distance between the mesial groove the first molars (MxIM)

- Mandible (Mn):

- Intercanine distance (MnIC)

- Distance between the buccal cusps of first premolars (MnIP)

- Distance between the distobuccal cusp of first molars (MnIM)

\section{Statistical analysis}

The statistical analysis was performed using Biostat 2009, Origin8 and Excel 2011. The normality of the data was checked with the Shapiro-Wilk's test. The Student's t-test was used to compare the normal data distribution of samples with the assumption of unequal variance (2 variables). ANOVA was employed to compare the means of three independent data sets that are normally distributed (ANB, SNA, SNB, MxIC, MnPM, MnIM). In case of finding a significant difference between groups (PP-MP), Bonferroni posthoc tests for repeated comparisons were performed to determine where the difference lies. Results are expressed as mean \pm SD.

For variables that did not show normal data distribution (SNMP, ArGoMe, MnIC, MxPM, MxIM), the non-parametric Kruskal-Wallis's test one-way analysis of variance was applied. Mann-Whitney ranking test was used to further examine the differences between groups when the assumption of normality was violated (ArGoMe, MxIM). The correlation and relationship between the parameters were evaluated by the Pearson correlation coefficient r. In case of fewer than 20 data, the Spearman's non-parametric test was applied with the R coefficient. The possible existence of non-linear relations was checked with the coefficient $\mathrm{R}$, or $\mathrm{R}^{2}$. Multivariate analysis was also performed and statistical significance was defined as $\mathrm{p}<0.05$.

\section{Results}

The values of measured skeletal and dental parameters are shown in Table 1.

\section{Comparison of skeletal values}

The statistical evaluation of ANB, SNA, SNB, SN-Ba, PP-MP and SN-MP angles showed no statistically significant differences between the study groups and controls $(p>0.05)$. A significantly increased ArGoMe angle ( $p=0.02$ ) was observed in MB patients compared with the controls. However, the ArGoMe angle of FMB patients showed no significant differences when compared with the control group $(\mathrm{p}=0.55)$. The results are graphically displayed in Figure 1.

\section{Comparison of dental values}

The MxIC, MnIC, MnIP, and MnIM widths showed no significant differences among the groups $(p>0.05)$. The statistical significance distinction of MxIP width of MB group remained on the borderline ( $p=0.07)$. When compared with the controls, the MxIM width of either the FMB group $(\mathrm{p}=0.01)$ or MB group $(\mathrm{p}=$ 0.00 ) showed a significant difference. The study groups presented 
Tab. 1. Values of examined skeletal and dental parameters.

\begin{tabular}{lccc}
\hline & NB & FMB & MB \\
\hline ANB & $4.0(2.3 ; 5.0)$ & $4.0(1.3 ; 6.0)$ & $4.3(3.0 ; 6.0)$ \\
SNA & $82.0 \pm 3.7$ & $81.1 \pm 4.3$ & $81.2 \pm 4.0$ \\
SNB & $76.7 \pm 3.7$ & $77.9 \pm 4.5$ & $78.3 \pm 3.8$ \\
SN-Ba & $129.4(126.8 ; 134.9)$ & $131.1(131.0 ; 132.4)$ & $130.3(127.6 ; 133.4)$ \\
PP-MP & $25.2 \pm 6.1$ & $24.0 \pm 5.7$ & $26.7 \pm 5.3$ \\
SN-MP & $31.0(26.8 ; 35.0)$ & $31.0(28.6 ; 35.8)$ & $33.0(28.8 ; 36.0)$ \\
ArGoMe & $123.9(119.3 ; 126.9)$ & $122.0(120.6 ; 129.4)$ & $126.7(122.8 ; 130.3)$ \\
MxIC & $33.7 \pm 2.1$ & $32.8 \pm 2.5$ & $34.4 \pm 2.4$ \\
MnIC & $26.3(25.4 ; 27.2)$ & $26.2(24.9 ; 27.3)$ & $26.3(24.9 ; 27.8)$ \\
MxIP & $41.0(39.3 ; 42.8)$ & $38.5(38.0 ; 42.8)$ & $39.9(37.8 ; 41.8)$ \\
MnIP & $33.5 \pm 1.9$ & $33.7 \pm 2.5$ & $33.6 \pm 2.0$ \\
MxIM & $46.6 \pm 2.7$ & $44.5 \pm 2.3$ & $44.6 \pm 3.0$ \\
MnIM & $47.2 \pm 2.6$ & $45.8 \pm 2.9$ & $47.1 \pm 3.1$ \\
\hline
\end{tabular}
for the mandible. Therefore, a tendency to class III is documented in the literature (15). In contrast, Pirilä-Parkkinen et al (16) support that mouth breathers acquire a skeletal class II because the individuals depress their mandible to keep their mouth open, which then exerts a backward pressure upon the ramus. As a result, the support of that mandible is displaced distally and its growth is usually retarded.

Contrary to the attention given to the sagittal and vertical axes, the transverse parameters have been inadequately investigated. However, in the literature, MB is mostly associated with a decreased maxillary width $(17,18)$. This may happen due to the lower position of the tongue, which inhibits the maintenance of the upper airway diameter (5).

Furthermore, adenoidectomy in children is reported to promote the normalization of skeletal characteristics in MB patients. Astudy of Swedish children showed that before their adenoidectomy, they had a significantly longer anterior face height and tendency towards maxillary compression, whereas, after their treatment, the results showed that on average, their dentofacial morphology was normalized and had a propensity for acquiring the mean values of the control group. More specifically, the five-year post-treatment follow up confirmed a shortening of the anterior cranial base and nose (19).

The present study is the first one to categorise 3 different groups of subjects according to their individual breathing pattern and medical history, as well as to test the effects of mouth breathing and adenoidectomy in comparison not only to nose breathers but also to each other.

More specifically, our FMB group consisted of former mouthbreathing patients who had been diagnosed with hypertrophy of adenoids and subsequently underwent adenoidectomy during childhood or 3 years before the day of admission to our department. The period of 3 years after surgery is considered to be adequate time for vertical skeletal parameters to normalise (20). As to the maxillary intermolar width, the results of our study showed a statistically significant distinction between the FMB and NB groups, which indicates a maxillary compression. Nevertheless, the transverse parameters of FMB and MB show no significant statistical change between them. As a result, the adenoidectomy is not effective in re-establishing the transverse parameters. In addition, no statistically significant difference was detected in the cephalometric parameters (vertical and sagittal) between FMB and NB individuals. Thus, the adjustment of nasal breathing with the 
removal of obstruction seems to facilitate the normalization of the tongue and hyoid bone postures, which accelerates the mandibular growth and closure of the mandibular plane angle.

The MB group was composed of patients with chronic nasal respiratory obstruction at the time of admission to the orthodontic department. This group showed a significantly decreased maxillary intermolar width in comparison to NB. However, the analysis of the rest of transverse parameters (MxIC, MxIP, MnIC, MnIP, MnIP) did not show any statistically significant difference among the groups. Besides, a substantial increase in the ArGoMe angle was observed, which indicates an increased mandibular clockwise rotation characterized by decreased posterior facial height and increased lower anterior facial height. The rest of cephalometric parameters displayed no abnormal growth changes. Moreover though, current studies still assume there is a connection between mouth breathing and skeletal malocclusions $(11,13)$; the absence of statistical difference in both ANB and SNB angles in our research indicates no tendency to a protruded/retruded position of the mandible in MB patients. Thus, a possible explanation of any tendency to class II/III found in literature could be based on genetic factors.

\section{Conclusion}

Until today, there is still not a commonly accepted protocol provided for the diagnosis of MB. In this study, the breathing mode was defined firstly by answers to questions listed in an extended questionnaire concerning the patients' medical history. and secondly, by their clinical examination. Thus, three different groups were formed regarding their breathing technique and adenoidectomy history.

The present study confirms the significant correlation between mouth breathing and vertical facial patterns with a decrease in maxillary intermolar width. The sagittal parameters did not show any statistically significant differences among the groups. As a result, it can be concluded that mouth breathing has less or even no influence on the sagittal growth and development of splanchnocranium. Due to the absence of statistically significant differences between the nose-breathing group and adenoidectomy group, it is confirmed that the removal of obstruction has a favourable effect on the vertical skeletal development of splanchnocranium. However, the transverse dental characteristics did not benefit since adenoidectomy patients showed a significant compression of maxilla.

\section{Learning points}

Mouth breathing is associated with long facial pattern and maxillary compression.

The sagittal parameters of mouth breathing patients remain unaffected.

Adenoidectomy has a favourable effect on the vertical skeletal growth and development of splanchnocranium, but not on transverse parameters.

\section{References}

1. McNamara JA Jr. Influence of respiratory pattern on craniofacial growth. Angle Orthod 1981; 51: 269-300.
2. Fricke HJ, Gebert R, Grabovski A, Hasund HG. Nasal airway, lip competence and craniofacial morphology. Eur J Orthodont 1993; 15: 297-304.

3. Solow B, Siersbaek-Nielsen S, Greve E. Airway adequacy, head posture and craniofacial morphology. Am J Orthodont 1984; 89: 214-223.

4. Pereira SR, Bakor SF, Weckx LL. Adenotonsillectomy in facial growing patients: Spontaneous dental effects. Braz J Otorhinolaryngol 2011; 77 (5): 600-614.

5. Arsalah R, Waheed H, Fatima J. Cephalometric assessment of patients with adenoidal faces. J Pak Med Assoc 2009; 59: 747-752.

6. Proffit WR. Contemporary Orthodontics and Dentofacial Orthopedics. Elsevier, 2018: 131-133.

7. Lofstrand-Tidestrom B, Hultcrantz E. Development of craniofacial and dental arch morphology in relation to sleep disordered breathing from 4 to 12 years. Effects of adenotonsillar surgery. Int J Pediatr Otorhinolaryngol 2010; 74: 137-143.

8. Zhu Y, Li J, Tang Y, et al. Dental arch dimensional changes after adenoidectomy or tonsillectomy in children with airway obstruction: A metaanalysis and systematic review under PRISMA guidelines. Medicine 2016; 95 (39): e4976.

9. Peltomaki T. The effect of mode of breathing on craniofacial growth. Eur J Orthod 2007; 29: 426-439.

10. Kluemper GT, Vig PS, Vig KW. Nasorespiratory characteristics and craniofacial morphology. The Eur J Orthodont 1995; 17 (6): 491-505.

11. Bianchini AP, Guedes ZC, Vieira MM. A study on the relationship between mouth breathing and facial morphological pattern. Braz J Otorhinolaryngol 2007; 73 (4): 500-515.

12. Dostalova T, Eliasova H, Gabcova D, Feberova J, Kaminek M. Contemporary skull development - palatal angle analysis. Bratisl Med J 2015; 116 (3): 143-146.

13. Zettergren-Wijk L, Forsberg CM, Linder-Aronson S. Changes in dentofacial morphology after adeno-/tonsillectomy in young children with obstructive sleep apnoea - a 5-year follow-up study. Eur J Orthod 2006; 28 (4): 319-326.

14. Rocha AC, Domínguez MEC, Reyes AD. Breathing mode influence on craniofacial development and head posture. J Pediatria 2018; 94 (2): 123-130.

15. Chung Leng Muñoz I, Beltri Orta P. Comparison of cephalometric patterns in mouth breathing and nose breathing children. Int J Pediatr Otorhinolaryngol 2014; 78 (7): 1167-1172.

16. Pirilä-Parkkinen K, Pirttiniemi P, Nieminen P, Tolonen U, Pelttari U, Löppönen H. Dental arch morphology in children with sleep-disordered breathing. Eur J Orthod 2009; 31 (2): 160-167.

17. Lione R, Franchi L, Huanca Ghislanzoni LT, Primozic J, Buongionno M, Cozza P. Palatal surface and volume in mouth-breathing subjects evaluated with three-dimensional analysis of digital dental casts-a controlled study. Eur J Orthod 2015; 37 (1): 101-114.

18. Malhotra S, Gupta V, Pandey RK, Singh SK, Nagar A. Dental consequences of mouth breathing in the pediatric age group. Int J Oral Health Sci 2013; 3: 79-83.

19. Lyle K. Airway compromise and dentofacial abnormalities. JGO 2000; 11: 9-18.

20. Zettergren-Wijk L, Forsberg CM, Linder-Aronson S. Changes in dentofacial morphology after adeno-/tonsillectomy in young children with obstructive sleep apnoea - a 5-year follow-up study. Eur J Orthod 2006; 28 (4): 319-326.

Received March 10, 2020. Accepted November 21, 2020. 